\title{
Temperature dependent total scattering structural study of $\mathrm{CaCu}_{3} \mathrm{Ti}_{4} \mathrm{O}_{12}$
}

\author{
E S Božin ${ }^{1}$, V Petkov ${ }^{2}$ P W Barnes ${ }^{3}$, P M Woodward ${ }^{3}$, T Vogt ${ }^{4}$, \\ S D Mahanti ${ }^{1}$ and S J L Billinge ${ }^{1}$ \\ ${ }^{1}$ Department of Physics and Astronomy and Center for Fundamental Materials Research, \\ Michigan State University, Biomedical Physical Sciences, East Lansing, MI 48824-2320, USA \\ 2 Physics Department, Central Michigan University, Mount Pleasant, MI 48859, USA \\ 3 Department of Chemistry, Ohio State University, Columbus, OH 43210-1185, USA \\ ${ }^{4}$ Physics Department, Brookhaven National Laboratory, Upton, NY 11973-5000, USA
}

Received 31 August 2004, in final form 2 September 2004

Published 22 October 2004

Online at stacks.iop.org/JPhysCM/16/S5091

doi:10.1088/0953-8984/16/44/007

\begin{abstract}
$\mathrm{X}$-ray and neutron powder diffraction data as a function of temperature are analysed for the colossal dielectric constant material $\mathrm{CaCu}_{3} \mathrm{Ti}_{4} \mathrm{O}_{12}$. The local structure is studied using atomic pair distribution function analysis. No evidence is found for enlarged oxygen or Ti displacement parameters suggesting that short range octahedral tilt disorder and off-centre Ti displacements are minimal. However, an unusual temperature dependence for the atomic displacement parameters of calcium and copper is observed. Temperature dependent modelling of the structure, using bond valence concepts, suggests that the calcium atoms become underbonded below approximately $260 \mathrm{~K}$, which provides a rationale for the unusually high $\mathrm{Ca}$ displacement parameters at low temperature.
\end{abstract}

\section{Introduction}

Recently there has been considerable interest in the dielectric properties of the cubic perovskiterelated $\mathrm{CaCu}_{3} \mathrm{Ti}_{4} \mathrm{O}_{12}(\mathrm{CCTO})$. This material exhibits a giant dielectric constant response with a highly unusual temperature dependence. It has a high and relatively temperature independent low frequency dielectric constant over a wide temperature range between 100 and $600 \mathrm{~K}$. However, below $100 \mathrm{~K}$ its value drops abruptly by almost three orders of magnitude, an effect that is not accompanied by a long range structural phase transition [1-3]. The crystal structure of CCTO (s.g. Im $\overline{3}$ ) [4] is shown in figure 1. Its structure can be derived from the ideal cubic perovskite structure by superimposing a body centred ordering of $\mathrm{Ca}$ and $\mathrm{Cu}$ ions and a pronounced tilting of the titanium centred octahedra (tilt system $\mathrm{a}^{+} \mathrm{a}^{+} \mathrm{a}^{+}$) [5]. The tilting dramatically alters the coordination environments of the A-site cations, leading to a 4-coordinate square-planar environment for $\mathrm{Cu}$ and a 12-coordinate icosahedral environment 


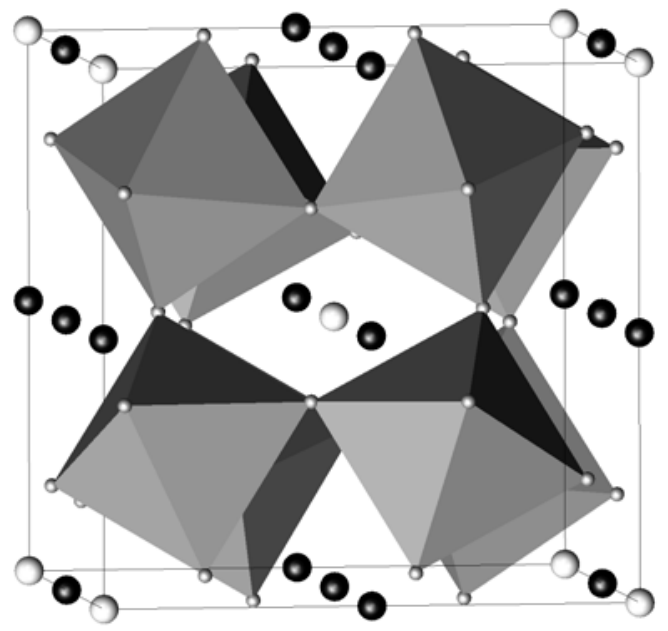

Figure 1. $\mathrm{CaCu}_{3} \mathrm{Ti}_{4} \mathrm{O}_{12}$ structure is cubic (space group Im $\overline{3}$ ): $\mathrm{TiO}_{6}$ octahedra, $\mathrm{Ca}$ atoms (light spheres), and $\mathrm{Cu}$ atoms (dark spheres).

for $\mathrm{Ca}$. It is the mismatch in size and bonding preferences of these two ions and the titanium that drives the large octahedral tilting distortion. The system is an antiferromagnetic insulator below $T_{\mathrm{N}}=25 \mathrm{~K}$, as indicated by transport, neutron diffraction, and Raman studies $[1,2,6]$.

Numerous proposals have been put forward for uncovering the origin of the giant dielectric response in $\mathrm{CCTO}$, including both intrinsic and extrinsic mechanisms. Intrinsic mechanisms comprise those referring to a lattice instability resulting in ferroelectric or relaxorlike behaviour, coupled with a highly correlated electronic ground state. Contrary to the case for other stoichiometric titanates, no ferroelectric transition has been observed in CCTO. Relaxor-like dynamical slowing down of dipolar fluctuations in nanosize domains has been proposed by Homes et al from optical conductivity measurements [3]. It has been argued that the high dielectric constant may arise from local dipole moments associated with offcentre displacements of Ti atoms [3]. The structure does not exhibit a long range structural distortion, as typically observed in perovskite compounds. Within this model the absence of a structural phase transition is explained by the premise that the tilts of the $\mathrm{TiO}_{6}$ octahedra are large enough to decouple the local titanium distortions from each other, effectively decoupling the ferroelectric order parameter and the long range crystal structure. The implications of this model could be tested experimentally using a local structural technique, such as the atomic pair distribution function (PDF) analysis used here. The PDF method utilizes both Bragg and diffuse intensities and yields the local atomic structure directly [7]. The off-centre displacements of Ti would imply large atomic displacement (thermal) parameters, and possibly octahedral tilt disorder.

Recently, Ramirez and collaborators suggested that local structural defects within the $\mathrm{Ca}-\mathrm{Cu}$ sublattice of CCTO, if evidenced, may provide an intrinsic scenario for explaining the gigantic dielectric constant observed in this system [8]. In this view, the defects would disrupt an otherwise rigid $\mathrm{Cu}-\mathrm{O}$ complex, and would be prone to local polarizability. However, since such defects are isolated and with low density, cooperative effects are absent. According to the authors, a significant diffuse scattering signal is expected in the high dielectric constant regime, with freezing at lower temperatures. Experimental verification of these predictions could also be performed by a local structural study such as the PDF method.

Another possible explanation for the colossal dielectric properties of CCTO is an extrinsic mechanism related to the microstructure of the sample, its morphology, and boundary layer 
effects [9]. Subramanian et al [1] argue that the unusually high dielectric constant of CCTO is due to its microstructure, because of the creation of an effective circuit of parallel capacitors as found in boundary layer dielectrics. A recent single-crystal x-ray diffraction study on CCTO single crystals showed twinning on a fine scale in the microstructure of CCTO [10]. Twin boundaries are proposed to act in such a manner as to create a barrier layer capacitance, an effect known to produce extremely high dielectric constants. The giant dielectric phenomenon has been attributed to a grain, or twin, boundary barrier layer capacitance [11, 12] where metallic grains are separated by non-percolating, insulating, twin or grain boundaries. Such a scenario (and the inverse case of insulating grains and conducting boundaries) has been tested theoretically and found to be in accord with experimental observations [13]. Finally, the colossal dielectric constant has also been attributed exclusively to the effects of depletion layers at the interface between the sample and measuring electrodes $[14,15]$. It appears clear that these extrinsic boundary layer effects play a role but it is controversial whether they, alone, account for the full colossal dielectric constant $[8,12,16]$.

In order to assess the validity of various explanations for the giant dielectric response of CCTO it is necessary to characterize not only the average crystal structure, but also the local structure. In this work local structural properties of the CCTO system are investigated as a function of temperature within a range from $50 \mathrm{~K}$ up to room temperature. Atomic pair distribution function analysis of the powder diffraction data has been employed to extract the local structural information [7].

\section{Experimental details}

The $\mathrm{CaCu}_{3} \mathrm{Ti}_{4} \mathrm{O}_{12}$ sample was prepared by conventional solid state methods. Stoichiometric amounts of $\mathrm{CaCO}_{3}$ (Mallinckrodt, $99.95 \%$ purity), $\mathrm{CuO}$ (J T Baker, 99.9\% purity), and $\mathrm{TiO}_{2}$ (EM Science, $99.7 \%$ purity) were weighed and mixed intimately using acetone in an agate mortar and pestle. The reaction mixture was preheated in a high form alumina crucible at $900^{\circ} \mathrm{C}$ for $8 \mathrm{~h}$. The preheated powder was reground and pressed into pellets. The pellets were heated in the presence of air at $1075^{\circ} \mathrm{C}$ for $24 \mathrm{~h}$. The purity of the final product was confirmed by x-ray powder diffraction using a Bruker D8 Advance diffractometer equipped with an incident beam Ge monochromator and a Braun position sensitive detector.

We performed a complementary neutron diffraction and x-ray diffraction study on powder samples of $\mathrm{CaCu}_{3} \mathrm{Ti}_{4} \mathrm{O}_{12}$. Time-of-flight neutron diffraction experiments were carried out at the Special Environment Powder Diffractometer (SEPD) at the Intense Pulsed Neutron Source (IPNS) at Argonne National Laboratory. About $10 \mathrm{~g}$ of polycrystalline sample was sealed in a cylindrical vanadium tube with He exchange gas. The sample was cooled using a closed-cycle He refrigerator, and data collected at 50, 100, 150, 200, and $290 \mathrm{~K}$. X-ray diffraction data were collected at the X7A beamline of the National Synchrotron Light Source (NSLS) at Brookhaven National Laboratory. The sample was carefully packed between Kapton foils to avoid texture formation, and mounted within a closed-cycle helium refrigerator. Complementary $\mathrm{x}$-ray PDF data were also collected at 50 and $300 \mathrm{~K}$ in symmetric transmission geometry using $29.08 \mathrm{keV}$ synchrotron radiation $(\lambda=0.4257 \AA)$. Scattered radiation was collected with an intrinsic germanium detector connected to a multichannel analyser. Several runs were conducted and the resulting x-ray diffraction patterns were averaged to improve the statistical accuracy and reduce any systematic effect due to instabilities in the experimental set-up. The raw data were background subtracted, corrected for flux and experimental effects such as sample absorption and multiple scattering, and normalized [17].

From the normalized data the total scattering structure function, $S(Q)$ [7], is obtained, where $Q=|\mathbf{Q}|=\left|\mathbf{k}-\mathbf{k}_{\mathbf{0}}\right|$ represents the momentum transfer (magnitude of the scattering 
vector). The PDF function, $G(r)$, is then obtained by a Fourier transformation according to

$$
G(r)=\frac{2}{\pi} \int_{0}^{\infty} Q[S(Q)-1] \sin (Q r) \mathrm{d} Q .
$$

The strength of the PDF technique is that it takes into account both Bragg and diffuse scattering components, and yields structural information on both long range order and local structural disorder in materials. The function $G(r)$ gives the number of atoms in a spherical shell of unit thickness at a distance $r$ from a reference atom. It peaks at characteristic distances separating pairs of atoms and thus reflects the atomic structure [17]. Neutron diffraction data processing was performed using the PDFgetN analysis program package [18]. The neutron PDFs examined in this paper use data over a wide momentum transfer range, and were terminated at $Q_{\max }=28 \AA^{-1}$. X-ray diffraction data were processed with the help of the programs RAD [19] and IFO [20]. The X-ray diffraction PDFs investigated here use data up to $Q_{\max }=24 \AA^{-1}$.

Structural information is obtained from the PDF data through a modelling procedure similar to the traditional reciprocal-space based Rietveld method performed by the GSAS program for example [21]. However, it is carried out in real space and yields the local structure rather than the average crystal structure [7, 17]. From the refinements, different structural information types can be extracted, such as lattice parameters, average atomic positions and amplitudes of their thermal motion, atomic displacements, and magnitudes of local octahedral tilts. The results reported here are obtained using the PDFFIT modelling program [22]. In cases where the average and local structure are the same (i.e., well-ordered crystals) the structural parameters obtained from Rietveld and PDF analysis are the same [23]. However, when the local structure deviates from the average structure the parameters from the PDF will reflect the local structure and the Rietveld parameters the periodically averaged values.

\section{Results}

First we compare our results with published structural data. Several studies of the average crystalline structure exist $[1,2,4,10]$. None of these studies finds evidence of a structural distortion as a function of temperature or pressure that would couple to the dielectric properties [8]. A systematic temperature dependent local structural study has not been performed. Local structure has only been investigated in related $\mathrm{CaCu}_{3} \mathrm{Ru}_{4} \mathrm{O}_{12}$, and in a different context [24]. Here we present a joint $\mathrm{X}$-ray and neutron diffraction study where the data are analysed both in real and in reciprocal space.

Typical Rietveld fits of the x-ray diffraction data are shown in figure 2 . The data are well explained within the $\operatorname{Im} \overline{3}$ space group at both low and high temperature, confirming the absence of phase transitions and distortions in the average structure, in quantitative agreement with earlier results [1].

Typical PDFs of CCTO both from neutron diffraction and from x-ray diffraction experiments are shown in figure 3. It should be noted that neutron PDFs of CCTO exhibit peculiar behaviour: contributions of $\mathrm{Ti}-\mathrm{O}$ and $\mathrm{Cu}-\mathrm{O}$ to the first PDF peak cancel out, so the first peak is 'missing'. This is due to the negative scattering length of Ti for neutrons, and due to almost identical component distances of $\mathrm{Ti}-\mathrm{O}$ and $\mathrm{Cu}-\mathrm{O}$. Inspecting the PDF profiles (figures 3(a) and (b)), one sees that the neutron scattering properties of Ti cause both positive and negative PDF peaks, i.e., peaks and valleys, respectively. This is in contrast to the case of $x$-ray diffraction PDFs where all the peaks contribute positive intensity (figure 3(c)). This perfect cancellation of the $\mathrm{Cu}-\mathrm{O}$ and $\mathrm{Ti}-\mathrm{O}$ intensities will only occur for selected peaks in the structure. Other peaks contain information about the relative positions of these ions and so a refinement over a wide range of $r$ will provide accurate structural information on these species. 


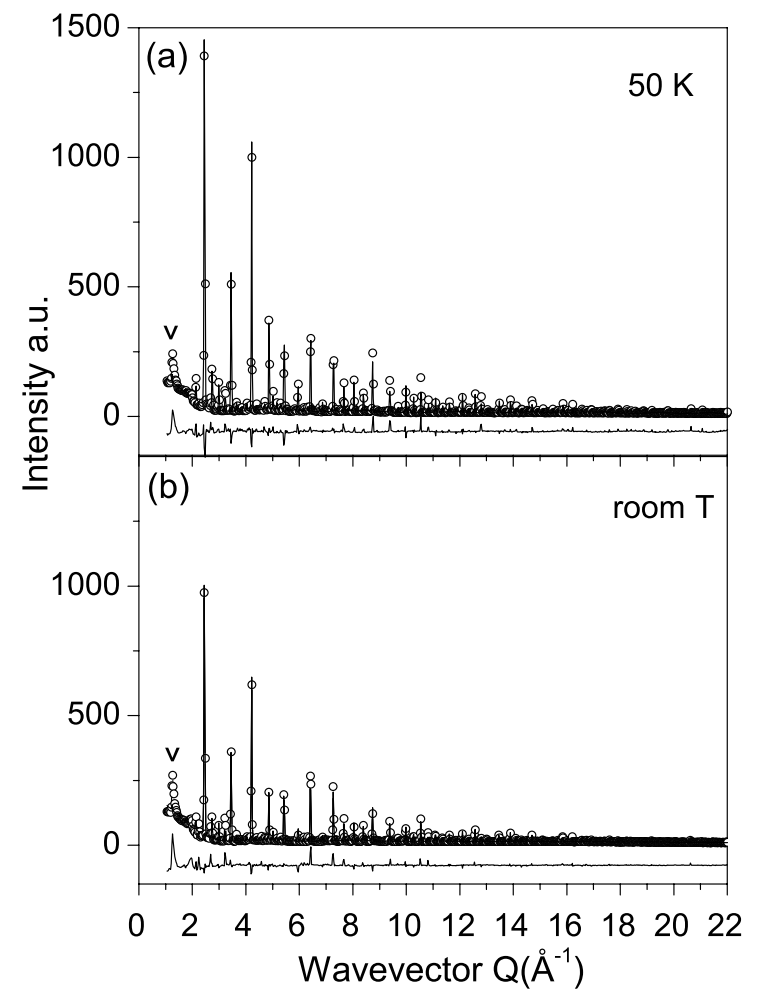

Figure 2. Experimental (open circles) and Rietveld fitted x-ray diffraction patterns (solid curve) of $\mathrm{CaCu}_{3} \mathrm{Ti}_{4} \mathrm{O}_{12}$ at (a) $50 \mathrm{~K}$ and (b) room temperature. The residual difference is given in the bottom part of each panel. The broad peak at very low values of $Q$ (marked with $\mathrm{V}$ ) originates from the sample environment (the aluminium shroud of the Displex cooling system).

Table 1. Results of the neutron powder diffraction structural study of CCTO, observing $\operatorname{Im} \overline{3}$ space group constraints: the lattice parameter and atomic coordinates. The atomic positions are $\mathrm{Ca}(0,0,0), \mathrm{Cu}(0,0.5,0.5), \mathrm{Ti}(0.25,0.25,0.25), \mathrm{O}(x, y, 0)$. Numbers in parentheses are expected standard deviations obtained from fitting.

\begin{tabular}{rlll}
\hline$T(\mathrm{~K})$ & $a(\AA)$ & $x(\mathrm{O})$ & $y(\mathrm{O})$ \\
\hline 50 & $7.3818(5)$ & $0.3029(1)$ & $0.1790(1)$ \\
100 & $7.3823(5)$ & $0.3029(1)$ & $0.1789(1)$ \\
150 & $7.3836(5)$ & $0.3031(1)$ & $0.1790(1)$ \\
200 & $7.3856(5)$ & $0.3031(1)$ & $0.1791(1)$ \\
290 & $7.3906(5)$ & $0.3032(1)$ & $0.1792(1)$ \\
\hline
\end{tabular}

Structural modelling of the CCTO PDF profiles was performed, observing constraints of the $\operatorname{Im} \overline{3}$ symmetry. The lattice parameter shows the expected temperature dependence. Structural parameters are summarized in table 1. Corresponding isotropic displacement parameters, $U_{\text {iso }}$, are listed in table 2. Nearest neighbour distances are shown in figure 4. While $\mathrm{Ca}-\mathrm{O}$ (solid triangles) and $\mathrm{Ti}-\mathrm{O}$ (solid squares) distances decrease monotonically with decrease in temperature, $\mathrm{Cu}-\mathrm{O}$ distance (solid circles) appears to be temperature independent, reflecting the rigidity of the square-planar coordination of $\mathrm{Cu}$ sublattice. Rietveld distances (not shown) are in agreement with the PDF within the experimental uncertainties. The distances at room temperature are in good agreement with those reported in [4], but differ somewhat 


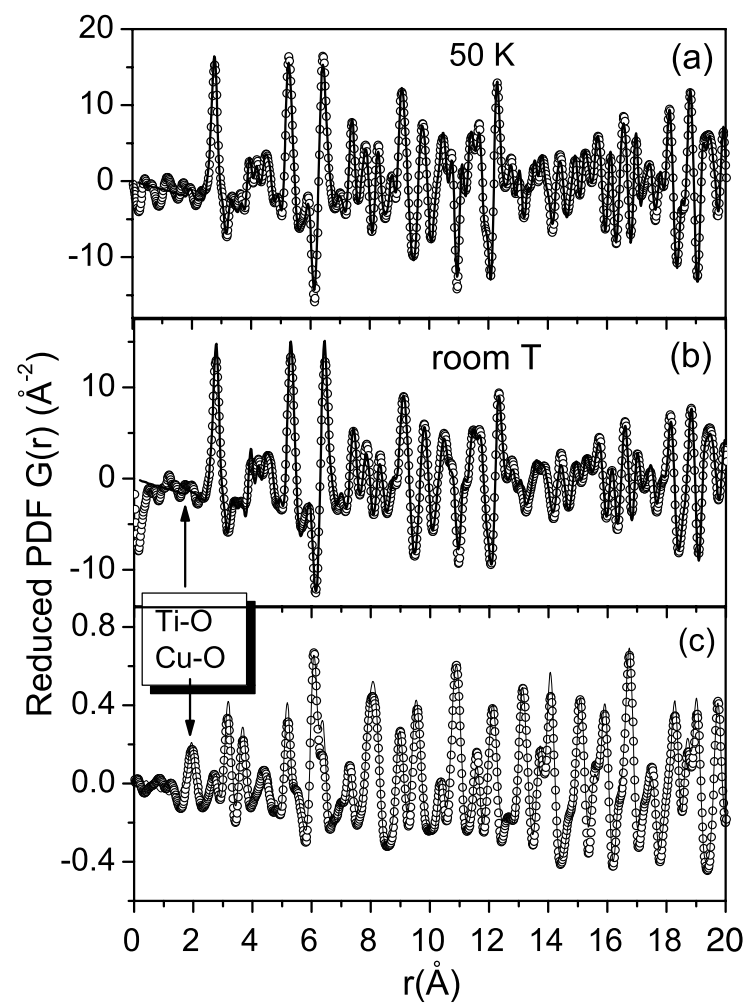

Figure 3. The reduced atomic PDF of CCTO. Experimental data (open circles) and fits (solid curves) are shown for: (a) data obtained from neutron diffraction at $50 \mathrm{~K}$ and (b) at room temperature; (c) data obtained from x-ray diffraction at room temperature. The goodness of the PDF fit is around $15 \%$. The PDF profiles corresponding to the two probes have different intensity distributions, reflecting different scattering properties of the sample for neutron diffraction and x-ray diffraction. Arrows mark the nearest neighbour PDF peak: in CCTO it contains two contributions, coming from $\mathrm{Ti}-\mathrm{O}$ and $\mathrm{Cu}-\mathrm{O}$ distances (see the text).

Table 2. Isotropic displacement parameters, $U_{\text {iso }}$, in units of $\AA^{2}$, as obtained from PDF structural refinements of neutron powder diffraction data. Numbers in parentheses are standard deviations estimated from fitting.

\begin{tabular}{rllll}
\hline$T(\mathrm{~K})$ & $\mathrm{Ca}$ & $\mathrm{Cu}$ & $\mathrm{Ti}$ & $\mathrm{O}$ \\
\hline 50 & $0.0041(2)$ & $0.0027(2)$ & $0.0028(2)$ & $0.0034(2)$ \\
100 & $0.0048(2)$ & $0.0028(2)$ & $0.0032(2)$ & $0.0038(2)$ \\
150 & $0.0055(2)$ & $0.0033(2)$ & $0.0034(2)$ & $0.0040(2)$ \\
200 & $0.0060(2)$ & $0.0042(2)$ & $0.0039(2)$ & $0.0043(2)$ \\
290 & $0.0057(2)$ & $0.0056(2)$ & $0.0047(2)$ & $0.0050(2)$ \\
\hline
\end{tabular}

from those reported in [1] where the temperature dependence of the $\mathrm{Cu}-\mathrm{O}$ bond lengths is more pronounced. The stronger scattering of oxygen in neutron data means that the current, temperature independent, $\mathrm{Cu}-\mathrm{O}$ distances are probably more reliable. As might be expected from the idea that copper is strongly covalently bonded with its four close neighbours, there is a much smaller dependence on $T$ for this parameter than for the other metal ions.

The results of an analysis of the $\mathrm{TiO}_{6}$ octahedra reveals very small changes in their volumes and shapes on cooling from room temperature down to $50 \mathrm{~K}$. Changes in the magnitude of the 


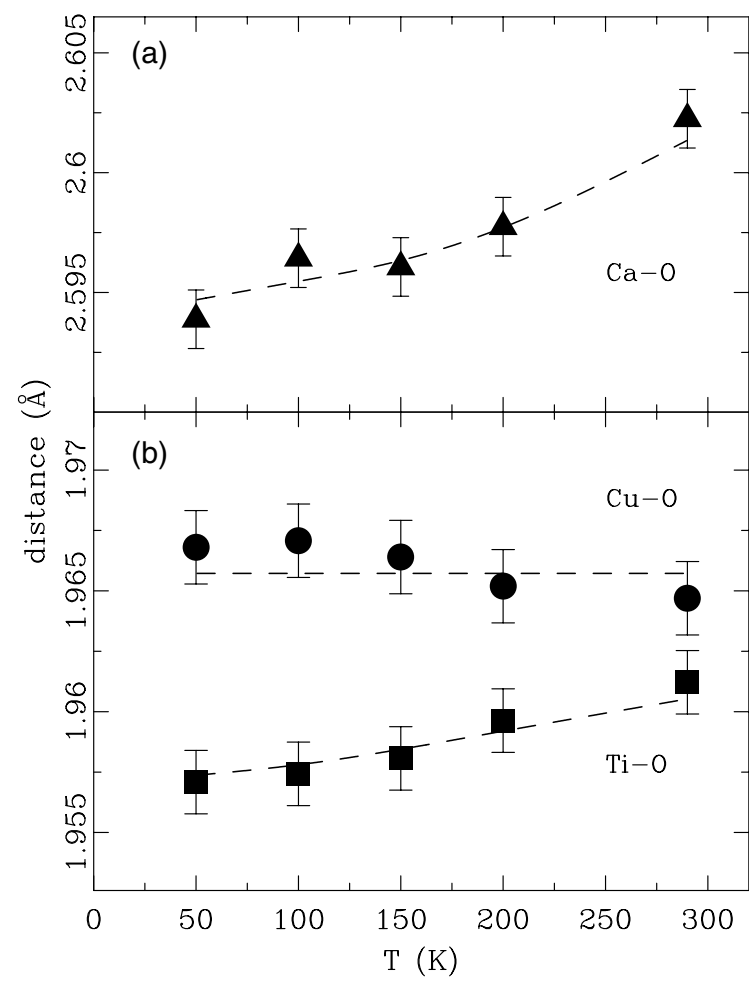

Figure 4. The temperature dependence of the nearest neighbour distances in $\mathrm{CaCu}_{3} \mathrm{Ti}_{4} \mathrm{O}_{12}$ : (a) $\mathrm{Ca}-\mathrm{O}$ (solid triangles), (b) $\mathrm{Cu}-\mathrm{O}$ (solid circles) and Ti-O (solid squares). Dashed curves are guides for the eye.

Table 3. Estimates of the Debye temperature, $\theta_{\mathrm{D}}$, and static offset parameter, $\sigma_{\mathrm{off}}^{2}$, for various constituent atoms of CCTO. Parameters are obtained by fitting the Debye model to the temperature dependences of the PDF displacement amplitudes.

\begin{tabular}{lll}
\hline Atom & $\Theta_{\mathrm{D}}(\mathrm{K})$ & $\sigma_{\text {off }}^{2}\left(\AA^{2}\right)$ \\
\hline $\mathrm{Ca}$ & $450(20)$ & $0.0021(1)$ \\
$\mathrm{Cu}$ & $360(20)$ & $0.0004(9)$ \\
$\mathrm{Ti}$ & $545(20)$ & $0.0014(2)$ \\
$\mathrm{O}$ & $923(20)$ & $0.0011(1)$ \\
\hline
\end{tabular}

average octahedral tilt angle are smaller than $0.3^{\circ}$ (less than $0.2 \%$ change). This agrees with the concept that the network of corner-shared octahedra is braced and made rigid by strong $\mathrm{Cu}-\mathrm{O}$ bonds [8]

Atomic displacement parameters for the constituent atoms are shown in figure 5. The data have been fitted with a Debye model in an effort to understand whether or not the low temperature thermal factors are anomalous [25-27]. Two-parameter fitting of the Debye curve to the displacement parameter temperature dependences has been carried out, using

$$
\sigma_{\mathrm{D}}^{2}=\frac{3 \hbar^{2}}{m k_{\mathrm{B}} \Theta_{\mathrm{D}}}\left[\frac{1}{4}+\left(\frac{T}{\Theta_{\mathrm{D}}}\right)^{2} \int_{0}^{\frac{T}{\Theta_{\mathrm{D}}}} \frac{x}{\mathrm{e}^{x}-1} \mathrm{~d} x\right]+\sigma_{\text {off }}^{2},
$$

where the parameters are the Debye temperature, $\Theta_{\mathrm{D}}$, and the static offset $\sigma_{\text {off }}^{2}$. Results of the fitting are summarized in table 3 and shown in figure 5 as the solid curves.

Oxygen and Ti parameters exhibit the expected behaviour with relatively high Debye temperatures and small offsets. The fits are shown in figure 5(b). The large Debye temperature of oxygen is similar to that obtained for cubic manganites [23], and is consistent with the 


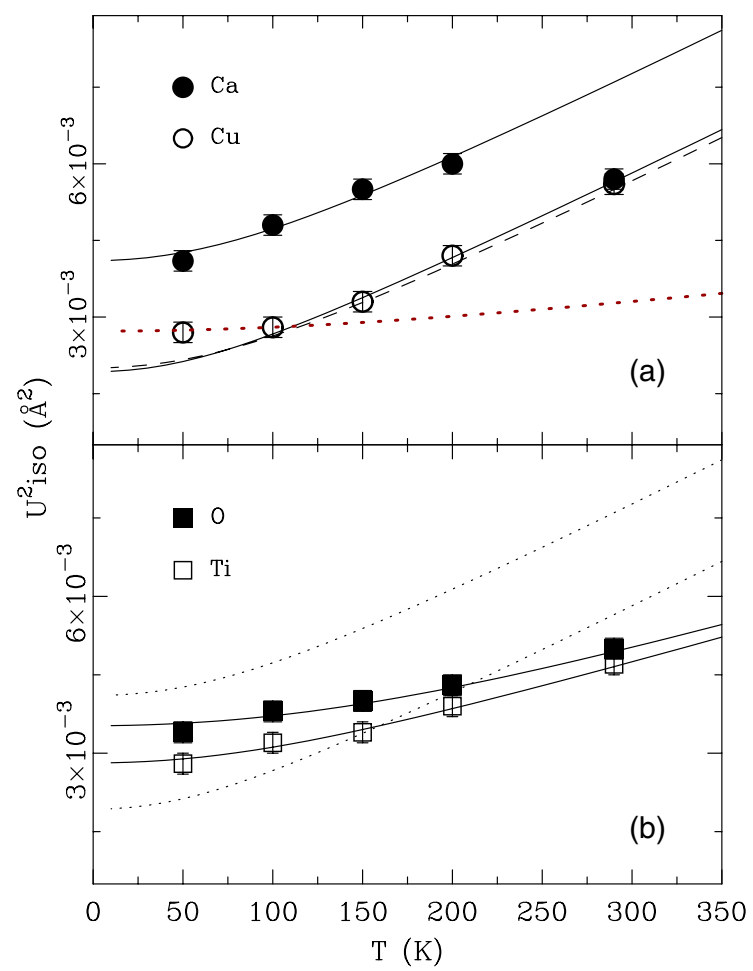

Figure 5. (a) Evolution with temperature of the isotropic displacement parameters of $\mathrm{Ca}$ and $\mathrm{Cu}$. Solid curves show the Debye model for $\mathrm{Ca}$ and $\mathrm{Cu}$, with parameters as specified in table 3 . The dashed curve is a Debye curve of Ca corresponding to the same Debye temperature, but with offset parameter set to zero (see the text). The thick dotted curve shows a Debye model for $\mathrm{Cu}$ using a higher Debye temperature of $785 \mathrm{~K}$. (b) Isotropic displacement parameters of $\mathrm{Ti}$ and $\mathrm{O}$ as a function of temperature, with Debye model curves superimposed (solid curves). Debye model curves for $\mathrm{Ca}$ and $\mathrm{Cu}$ (dotted curves) are also shown for comparison.

(This figure is in colour only in the electronic version)

observed stiffness of the $\mathrm{Cu}-\mathrm{O}$ complex. The Debye parameters for $\mathrm{Ti}$ are also similar to those for perovskite titanates such as $\mathrm{BaTiO}_{3}$, for example [28]. There is, therefore, no evidence for local disorder on the titanium or oxygen sublattices. This suggests that neither nanoscale tilt disorder [8] nor significant off-centre Ti displacements [3] are present to explain the colossal dielectric behaviour.

The $\mathrm{Ca}$ and $\mathrm{Cu}$ parameters behave more interestingly. The atomic displacement parameter associated with the $\mathrm{Ca}$ sites remains unusually large at low temperature (almost twice as high as that of $\mathrm{Cu}$ ) with a significant offset parameter. This may suggest local static displacive disorder on this site, with a magnitude of approximately $0.05 \AA$. This will be discussed later in terms of bond valence sum predictions for the Ca site. A single point at high temperature lies below the line. Further confirmation of whether this is a real effect is needed.

The dashed curve in figure 5(a) is the same Debye curve of Ca, but with the offset parameter set to zero. For this curve it is interesting to note that the high temperature $U_{\text {iso }}(\mathrm{Ca})$ point lies on the dashed curve where the static component is switched off. This observation may be suggestive of a crossover from a disordered state at low temperature to a more ordered state at high temperature, with a crossover temperature in the 200-300 K range. 


\section{Discussion}

\subsection{Bond valence sum prediction of the crystal structure of CCTO}

Here we discuss bond valence sum calculations that can be used to make predictions about the stability as a function of temperature of the observed cubic structure. These may indicate a tendency to local structural distortions that are prevented from propagating by the bracing described above.

Normally in an $\mathrm{ABO}_{3}$ perovskite (e.g. $\mathrm{CaTiO}_{3}$ ) the octahedral tilting distortion increases as the temperature decreases. This occurs because the ionic A-O bonds contract more rapidly in response to the decreasing temperature than the more covalent $\mathrm{B}-\mathrm{O}$ bonds. However, in CCTO the situation is somewhat different. Firstly, the $\mathrm{Cu}-\mathrm{O}$ bond length is very sensitive to changes in the magnitude of the tilting distortion, whereas the $\mathrm{Ca}-\mathrm{O}$ bond length contracts much more slowly with increasing tilt. Secondly, the $\mathrm{Cu}-\mathrm{O}$ bonds are considerably more covalent than the typical A-O bond, and exhibit relatively little thermal expansion over the temperature range examined in this study, as shown in figure 4. These considerations help to explain why both the tilt magnitude and the $\mathrm{Cu}-\mathrm{O}$ distance are essentially independent of temperature.

As described above, the $\mathrm{Cu}-\mathrm{O}$ bonding locks in the tilting at what is essentially a constant value. However, the optimal $\mathrm{Ca}-\mathrm{O}$ bond length should still contract fairly substantially with decreasing temperature, as it would in a typical perovskite such as $\mathrm{CaTiO}_{3}$. Consequently, there will be a crossover temperature where the $\mathrm{Ca}-\mathrm{O}$ and $\mathrm{Cu}-\mathrm{O}$ bonding are perfectly matched. Above this temperature the calcium will be overbonded. That is to say, the effective size of $\mathrm{Ca}$ will be too large for its environment and the $\mathrm{Ca}-\mathrm{O}$ bonds will be in compression. Below the optimal temperature the calcium will be underbonded, so the effective size of $\mathrm{Ca}$ is too small for its environment and the $\mathrm{Ca}-\mathrm{O}$ bonds will be in tension. In order to estimate the optimal temperature for CCTO we have utilized the structure prediction capabilities of the SPuDS software package [29] to evaluate the competition between $\mathrm{Cu}-\mathrm{O}, \mathrm{Ca}-\mathrm{O}$, and Ti-O bonding as a function of temperature. SPuDS is based on the bond valence concept $[30,31]$ and has been shown to be quite accurate in predicting the magnitude of octahedral tilting distortions in perovskites [29]. The thermal expansion of all bonds was assumed to be inversely related to the valence of the bond as suggested by Brown [32]. Figure 6 shows the predicted Ti-O-Ti angles as a function of temperature for $\mathrm{CaCu}_{3} \mathrm{Ti}_{4} \mathrm{O}_{12}$ and $\mathrm{CaTiO}_{3}$. In agreement with the experimental results reported here, the temperature dependence of the tilting in CCTO is predicted to be much smaller than that of $\mathrm{CaTiO}_{3}$. The predicted calcium and copper bond valence sums, as well as the global instability index [33], are shown in figure 7 as a function of temperature. The global instability index reaches a minimum near $260 \mathrm{~K}$, where the bonding requirements of calcium and copper are optimally matched; the bond valence sum calculations indicate that the structure is most stable at this point. Below this temperature the $\mathrm{Ca}$ atoms become underbonded and the copper atoms overbonded. Underbonded atoms tend to move off-centre in their coordination polyhedron [31]. This might explain the significant static offset needed in the Debye fits to the Ca ions. If the off-centre $\mathrm{Ca}$ displacements are locally correlated, this will result in local ferroelectric domains and an enhanced intrinsic dielectric constant at low $T$.

\subsection{Implications for dielectric properties}

We address possible implications for the dielectric properties of CCTO of the disorder on the Ca sublattice suggested by the PDF results. Although there is now mounting evidence that the unusual dielectric properties of CCTO may be extrinsic in nature [10,14], the possibility for an 

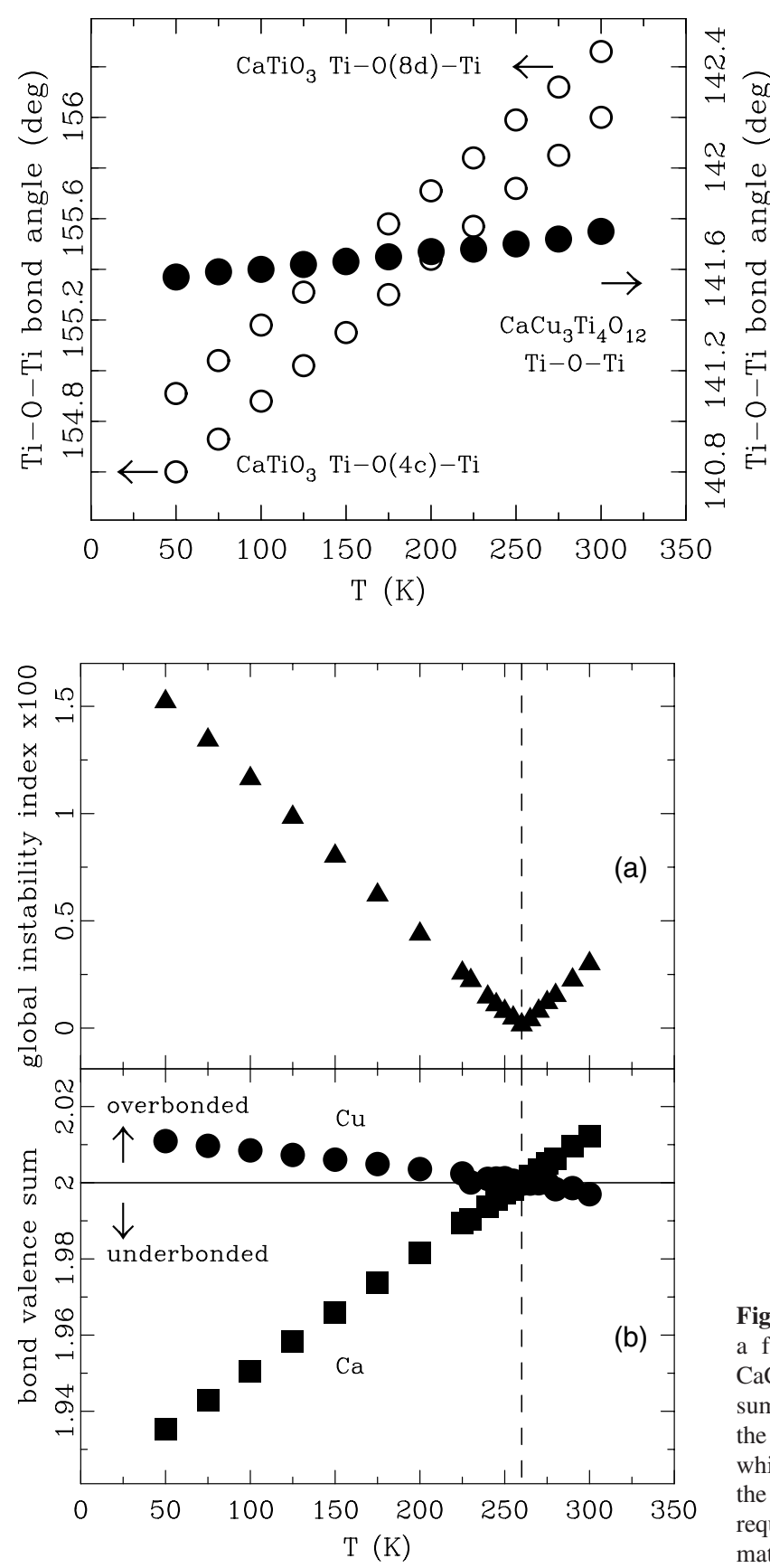

Figure 6. Expected $\mathrm{Ti}-\mathrm{O}-\mathrm{Ti}$ bond angles as a function of temperature for $\mathrm{CaCu}_{3} \mathrm{Ti}_{4} \mathrm{O}_{12}$ and $\mathrm{CaTiO}_{3}$.

Figure 7. (a) Global instability index as a function of temperature, calculated for $\mathrm{CaCu}_{3} \mathrm{Ti}_{4} \mathrm{O}_{12}$. (b) Predicted bond valence sums for $\mathrm{Ca}$ and $\mathrm{Cu}$. The solid line denotes the optimal valence for the two atomic types, while the dashed line marks the minimum of the global instability index where the bonding requirements of $\mathrm{Ca}$ and $\mathrm{Cu}$ are optimally matched.

intrinsic mechanism is not completely ruled out [8]. One of the remaining plausible avenues for the intrinsic mechanism involves a system that is at the edge of the Clausius-Mossotti catastrophe [14]. The origin of the gigantic dielectric constant in the low frequency mode and also that of the unusually large dielectric constant in the high frequency regime (of the order of 100), as well as the exact nature of the mechanism of switching between these two, are not clearly understood. 
The defect model in [8] describes a system involving a small concentration of polarizable defects, with a temperature independent polarization, embedded in a region with the background dielectric constant, $\epsilon_{0}$ [8]. The enormous real part of the dielectric constant of such a system is then readily achieved provided that (i) $\epsilon_{0} \gg 1$, (ii) the polarizability of the defects is of the order of their volume, and (iii) the concentration of the defects is reasonably small, $\ll 1$. This model appears to capture rather nicely the unusually high value of the measured dielectric constant plateau, of the order of 5000 [8]. However, the sensitivity of the dielectric constant plateau value, within this model, to the exact value of $\epsilon_{0}$ used is remarkable. Changing $\epsilon_{0}$ from 100 to about 70, and keeping all other parameters in the model the same, changes the plateau value from about 5128 to only about 223 . This demonstrates the importance of the background dielectric constant within this model [34].

Study of the optical properties of CCTO [3] reveals an unusual temperature dependence of the real part of the dielectric function in the far infrared (FIR) limit $\left(\omega \leqslant 20 \mathrm{~cm}^{-1}\right), \epsilon_{\mathrm{FIR}}$. This is found to change from $\sim 70$ at high temperature to $\sim 120$ at the base temperature, an increase of about $70 \%$. The order of $\epsilon_{\mathrm{FIR}}$ was concluded to be in good agreement with the high frequency limit (and low temperature) value of the measured dielectric constant of CCTO; presumably $\epsilon_{0}$ featured in the defect model.

While the issues addressed here are far from being resolved, we speculate that the $\mathrm{Ca}$ sublattice may play a key role in explaining some of the observed properties of CCTO. Distribution of the $\mathrm{Ca}$ atoms between displaced lattice sites in some sort of multi-well potential at low temperatures may provide a natural explanation for the observed enlarged thermal amplitudes. Furthermore, if this happens in a locally correlated fashion, within some sort of nanodomain structure, it could possibly be relevant for the anomalous increase of far infrared $\epsilon_{\mathrm{FIR}}$ observed in studies of the optical properties [3]. In this view, upon increasing the temperature, the local order on Ca sites is destroyed, and this leads to a decrease of the intrinsic (high frequency) dielectric constant.

\section{Conclusions}

Local structural properties of the CCTO system are investigated as a function of temperature within a range from $50 \mathrm{~K}$ up to room temperature. Data were analysed using Rietveld refinement and the atomic pair distribution function (PDF) analysis of the powder diffraction data has been employed to extract the local structural information. In agreement with earlier studies, no signature of a phase change has been observed. The temperature dependence of the nearest neighbour $\mathrm{Cu}-\mathrm{O}$ distance is very flat, supporting the idea that this is bracing the structure. No evidence for nanoscale octahedral tilting, or off-centre Ti displacements, is found. However, the isotropic $\mathrm{Ca}$ displacement parameter has a significant static offset compared to the expected Debye behaviour.

Simple modelling based on bond valence concepts supports the local structural observations. The temperature at which both the $\mathrm{Ca}$ and $\mathrm{Cu}$ environments are optimal has been estimated to be in the vicinity of $260 \mathrm{~K}$ and at lower temperatures the $\mathrm{Ca}$ becomes underbonded. This is generally accompanied by a reduction in site symmetry and may result in an off-centre displacement of $\mathrm{Ca}$ ions. If these displacements cannot become long range ordered because of the braced structure, this might explain the absence of a structural phase transition and the static offset of the $\mathrm{Ca}$ thermal parameters.

Local domains of coherently polarized displaced $\mathrm{Ca}$ ions could possibly exist at low temperature and may be related to the enhanced values of far IR lattice dielectric constant, a parameter playing an important role in intrinsic [8] and intrinsic boundary layer capacitance $[12,13]$ models for the large dielectric response. 


\section{Acknowledgments}

We are grateful to Simine Short for providing valuable help with the neutron diffraction data collection. This work was supported by DOE-BES through grant FG02-97ER45651. The experimental data were collected on the X7A beamline at the NSLS at Brookhaven National Laboratory and on SEPD at the IPNS at Argonne National Laboratory. NSLS is funded by the US Department of Energy under contract DE-AC02-98CH10886. IPNS is funded by the US Department of Energy under contract W-31-109-ENG-38.

\section{References}

[1] Subramanian M A, Li D, Duan N, Reisner B A and Sleight A W 2000 J. Solid State Chem. 151323

[2] Ramirez A P, Subramanian M A, Gardel M, Blumberg G, Li D, Vogt T and Shapiro S 2000 Solid State Commun. 115217

[3] Homes C C, Vogt T, Shapiro S M, Wakimoto S and Ramirez A P 2001 Science 293673

[4] Bochu B, Deschizeaux M N, Joubert J C, Collomb A, Chenavas J and Marezio M 1979 J. Solid State Chem. 29 291

[5] Glazer A M 1972 Acta Crystallogr. B 283384

[6] Koitzsch A, Blumberg G, Gozar A, Dennis B, Ramirez A P, Trebst S and Wakimoto S 2002 Phys. Rev. B 65 052406

[7] Egami T and Billinge S J L 2003 Underneath the Bragg Peaks: Structural Analysis of Complex Materials (Oxford: Pergamon)

[8] Ramirez A P, Lawes G, Butko V, Subramanian M A and Varma C M 2002 Preprint cond-mat/0209498

[9] He L, Neaton J B, Cohen M H, Vanderbilt D and Homes C C 2002 Phys. Rev. B 65214112

[10] Subramanian M A and Sleight A W 2002 Solid State Sci. 4347

[11] Sinclair D C, Adams T B, Morrison F D and West A R 2002 Appl. Phys. Lett. 802153

[12] Homes C C, Vogt T, Shapiro S M, Wakimoto S, Subramanian M A and Ramirez A P 2003 Phys. Rev. B 67 092106

[13] Cohen M H, Neaton J B, He L and Vanderbilt D 2003 J. Appl. Phys. 943299

[14] Lunkenheimer P, Bobnar V, Pronin A V, Ritus A I, Volkov A A and Loidl A 2002 Phys. Rev. B 66052105

[15] Lunkenheimer P, Fichtl R, Ebbinghaus S G and Loidl A 2004 Preprint cond-mat/0403119

[16] Kobayasi W and Terasaki I 2003 Physica B 329771

[17] Billinge S J L 1998 Local Structure from Diffraction ed S J L Billinge and M F Thorpe (New York: Plenum) p 137

[18] Peterson P F, Gutmann M, Proffen Th and Billinge S J L 2000 J. Appl. Crystallogr. 331192

[19] Petkov V 1989 J. Appl. Crystallogr. 22387

[20] Petkov V and Danev R 1998 J. Appl. Crystallogr. 31609

[21] Larson A C and Von Dreele R B 1994 Report No LAUR-86-748, Los Alamos National Laboratory

[22] Proffen Th and Billinge S J L 1999 J. Appl. Crystallogr. 32572

[23] Proffen Th, DiFrancesco R G, Billinge S J L, Brosha E L and Kwei G H 1999 Phys. Rev. B 609973

[24] Ebbinghaus S G, Weidenkaff A and Cava R J 2002 Solid State Commun. 167126

[25] Kittel C 1963 Quantum Theory of Solids (New York: Wiley)

[26] Beni G and Platzman P M 1976 Phys. Rev. B 141514

[27] Jeong I-K, Heffner R H, Graf M J and Billinge S J L 2002 Preprint cond-mat/0209603

[28] Kwei G H, Lawson A C, Billinge S J L and Cheong S-W 1993 J. Phys. Chem. 972368

[29] Lufaso M W and Woodward P M 2001 Acta Crystallogr. B 57725

[30] Brown I 1978 Chem. Soc. Rev. 7359

[31] Brown I D and Altermatt D 1985 Acta Crystallogr. B 41244

[32] Brown I D, Dabkowski A and McCleary A 1997 Acta Crystallogr. B 53750

[33] Salinas-Sanchez A, Garcia-Munoz J L, Rodriguez-Carvajal J, Saez-Puche R and Martinez J L 1992 J. Solid State Chem. 100201

[34] Bozin E S, Mahanti S D and Billinge S J L, unpublished 